\title{
SUPERNOVAE IN MASSIVE INTERACTING BINARIES
}

\author{
P. C. JOSS ${ }^{1}$, J. J. L. HSU ${ }^{1,2}$, PH. PODSIADLOWSKI ${ }^{3}$, R. R. ROSS ${ }^{4}$ \\ 1 M. I. T, Cambridge, MA 02139, U.S.A. \\ 2 University of California, Berkeley, CA 94720, U.S.A. \\ 3 Institute of Astronomy, Cambridge CB3 OHA, U.K. \\ 4 College of the Holy Cross, Worcester, MA 01610, U.S.A.
}

\begin{abstract}
We here report the results of a systematic investigation of how binary interaction affects the presupernova evolution of massive stars and the resulting supernova explosions. We summarize the various types of binary interaction and the evolutionary scenarios in which they are realized. We also present the results of a series of hydrodynamical calculations which model the supernova explosion for various progenitor types and discuss their observable characteristics.
\end{abstract}

\section{The Effects of Binary Interaction}

In general, binary interaction can affect the structure of a massive star in three fundamentally different ways: by mass loss, mass accretion or common-envelope evolution (for details and references, see Podsiadlowski, Joss, and Hsu 1992).

\subsection{MASS LOSS}

At least $20 \%$ of all massive stars are expected to experience mass loss via Roche-lobe overflow to close binary companions during their lifetimes. In most cases, these stars will lose all of their hydrogen-rich envelopes and become helium stars, which are good candidates for type Ib supernovae. In some cases, when mass transfer is non-dynamical and when the binary components are close in mass, the primary can retain part of its hydrogen-rich envelope (although at most a few solar masses). The resulting supernova explosions of these progenitors (which we call type II [stripped]) are distinct from the majority of type II supernovae (see 3.1.).

\subsection{MASS ACCRETION}

Mass accretion (generally onto the secondary) occurs in most mass-transfer scenarios (except in cases where mass transfer is dynamical). The post-accretion evolution depends on whether the secondary has already left the main sequence or is still burning hydrogen in its core. If mass transfer occurs when the secondary is still on the main sequence, the secondary will be rejuvenated as a main-sequence star and subsequently mimic the standard evolution of a more massive single star. If it has already left the main sequence, the subsequent evolution can be drastically different. In particular, it may end its life as a blue supergiant rather than as a red supergiant. The resulting supernova (which we call type II [blue]) will resemble SN 1987A (see 3.2.). 


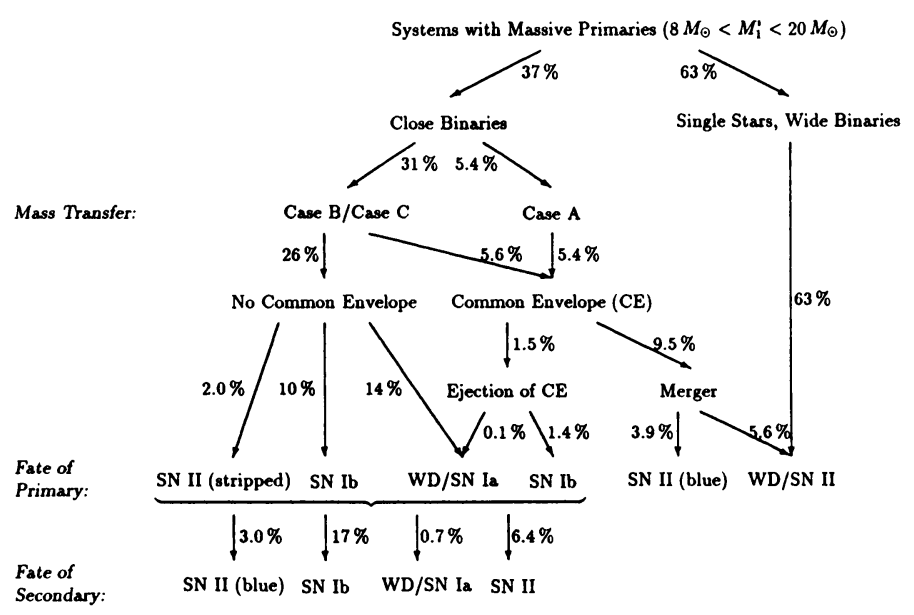

Figure 1. Summary of evolutionary scenarios for systems containing massive primaries with masses between $8 M_{\odot}$ and $20 M_{\odot}$ (simplified). The percentages give the (theoretical) probabilities that a system with a primary in the chosen mass range passes through the particular evolutionary channel. (The probabilities are based on Monte-Carlo simulations and are only very approximate.)

\subsection{COMMON-ENVELOPE EVOLUTION}

The most dramatic type of binary interaction is the merger of two stars in a common envelope. If the common envelope is ejected, the outcome will be a short-period helium-star (or degeneratedwarf) binary, possibly leading to a type I supernova. If the two stars merge completely (which is likely to happen when the initial binary period is relatively short and the common envelope tightly bound), the end product will be a single, massive star with some highly unusual properties: it will be rapidly rotating, probably have a thoroughly mixed envelope and may end its evolution as a blue supergiant, i.e. in a type II [blue] supernova (this depends on the relative mass fraction in the envelope and the helium abundance in the envelope).

\section{Evolutionary Scenarios and Monte Carlo Simulations}

Figure 1 provides an overview of evolutionary scenarios in which these various possibilities are realized. The diagram is simplified and contains only the most important channels. The percentages given next to each channel are based on Monte Carlo simulations and are very uncertain (due to numerous uncertainties caused by incomplete statistical data and our poor understanding of some of the major phases of binary evolution [common-envelope evolution etc.]). The main conclusions of our Monte Carlo study are:

- The evolution of a large fraction ( $20-35 \%$, assuming a binary frequency of $1 / 3-2 / 3)$ of all massive stars is substantially affected by binary interaction. In most cases, the resulting supernova explosions differ substantially from ordinary type II supernovae.

- Some $15-30 \%$ of all massive stars are expected to lose all of their hydrogen-rich envelopes.

- In a significant fraction of cases $(\sim 5 \%)$, binary evolution will lead to a blue supergiant with a substantial hydrogen-rich envelope. The resulting supernova explosion will resemble SN 1987A.

- In a limited parameter regime ( $1-2 \%$ of the cases considered) is it possible for a massive primary to retain part of its hydrogen-rich envelope after a mass-loss event. 


\section{Hydrodynamical Calculations}

For our hydrodynamical calculations we employed a one-dimensional, explicit code, which is conservative in the fluxes of energy and momentum. We included flux-limited diffusion, an equation of state allowing partial ionization of hydrogen and helium, and the heating due to nickel and cobalt decay. (For a detailed description of the code and the calculations see Hsu et al. 1992.)

\subsection{RED SUPERGIANT PROGENITORS WITH MASS LOSS}

We have calculated the explosion of four red giants with a core mass of $3.2 M_{\odot}$ and various envelope masses $\left(0.5,2,5\right.$ and $\left.8.8 M_{\odot}\right)$. Our main conclusions are:

- As the mass of the ejecta increases, the light curve of the supernova appears to shift gradually from a linear shape to one with a plateau (see figure 2).

- Given the same explosion energy, the energy available per unit mass for the stars with severe mass loss is larger, thus the overall velocity higher. In particular, the velocity of the material in the deep interior is significantly lower for a larger ejecta mass.

- At early stages, a small ejecta mass or a large explosion energy results in initially higher photospheric velocities. However, these cases also result in lower photospheric velocities at late times, as the gas cools and the photosphere recedes inward.

\subsection{BLUE SUPERGIANT PROGENITORS}

A significant fraction of stars explode as blue supergiants because of a previous binary interaction. The resulting supernova explosions will resemble SN 1987A, and many of the results of the modeling of SN 1987A can be applied directly to this supernova type (type II [blue]). However, in general the compactness of the progenitor will depend on the amount of mass that has been accreted (and possibly on the amount of mixing induced as a result). In figure 3, we compare the light curve of a blue supergiant (a $17 M_{\odot}$ star that accreted $3 M_{\odot}$ after its main-sequence phase) with the light curve of a $20 M_{\odot}$ red supergiant without mass loss. The light curve of the blue star is initially (before day 10) underluminous by $\sim 1 \mathrm{mag}$ (because of its smaller radius), and by $\sim 3 \mathrm{mag}$ (between day $\sim 15-70$ ). Blue stars have larger initial photospheric velocities, and the ejecta expand and cool more quickly. As a consequence, the real size of the ejecta for the explosion of the blue star is almost always larger than that for the red star, while the size of the photosphere is almost always smaller. The escape of $\mathrm{Ni} / \mathrm{Co}$ decay energy $\left(0.1 M_{\odot}\right.$ for the blue star in fig. 3$)$ causes a later rise in the light curve (no mixing of radioactive material into the outer layers was assumed in these models).

\section{Conclusions and Outlook}

We have shown that the structure of a large fraction of all massive supernova progenitors stars is affected by a previous binary interaction and that, in most cases, the resulting supernova explosions will be significantly different from standard type II explosions. The next step (which we pursue at present) is to devise simple diagnostics which one can use to compare these various supernova types with observed supernovae. Only when this has been achieved can we hope to understand the final phase in the evolution of massive stars.

\section{References}

Hsu, J. J. L, Ross, R. R., Joss, P. C., and Podsiadlowski Ph. (1992), in preparation.

Podsiadlowski, Ph., Joss, P. C., Hsu, J. J. L. (1992), in press. 


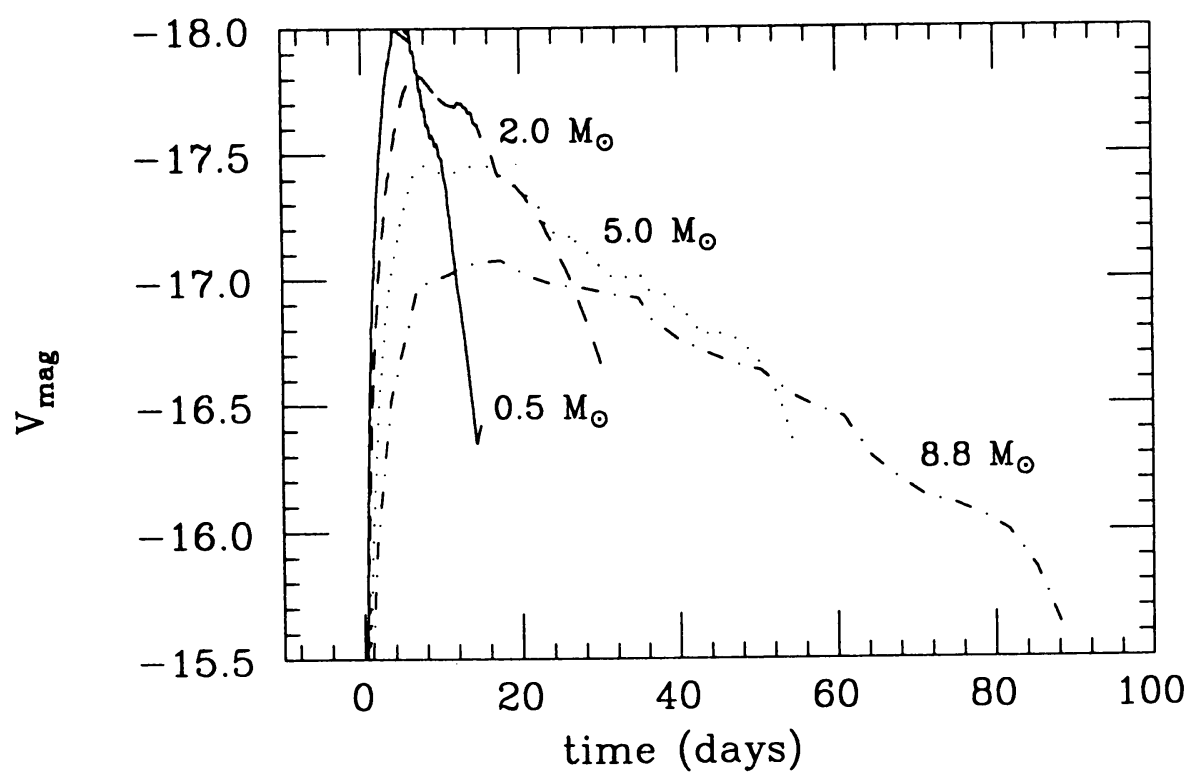

Figure 2. The optical light curves for a series of red giant progenitors (with a core mass of $3.2 M_{\odot}$, and hydrogen-rich envelope masses of $0.5,2,5$ and $8.8 M_{\odot}$, respectively). The explosion energy was $10^{51} \mathrm{erg}$ in all calculations. The morphology of the light curve changes systematically from type II [L] to type II [P] with increasing envelope mass. The stripped giant (with an envelope mass of $0.5 M_{\odot}$ ) rapidly reaches its maximum photospheric velocity, which then decreases rapidly.

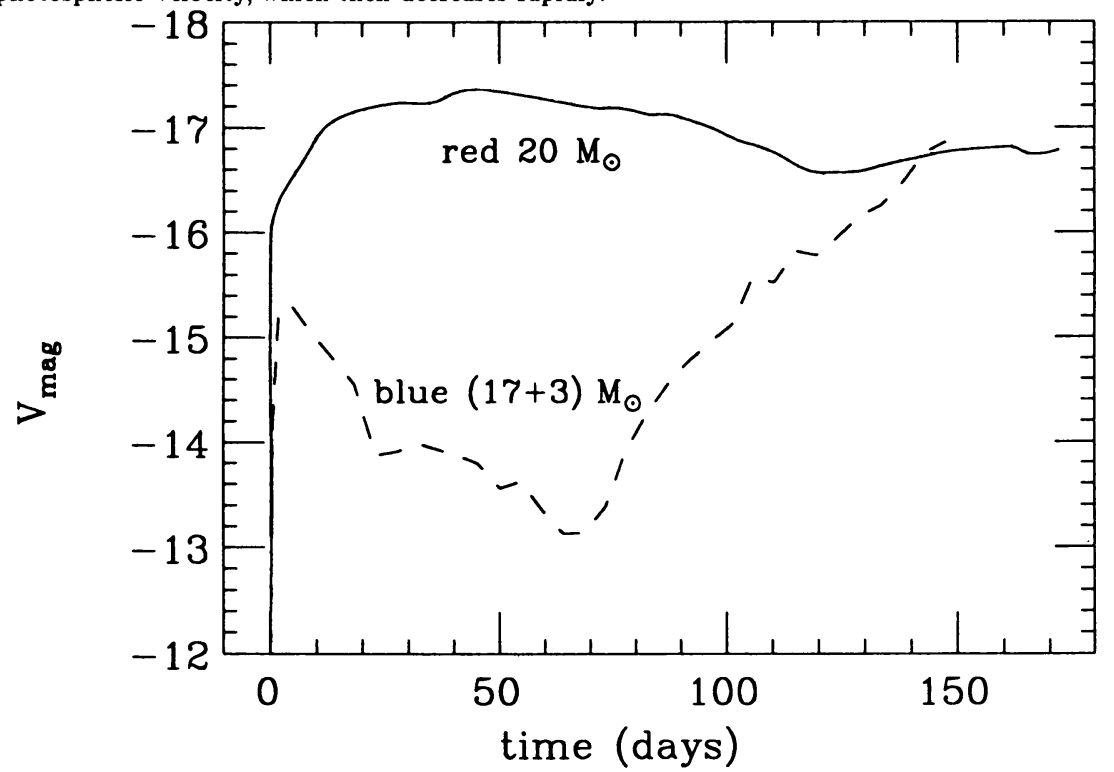

Figure 3. The optical light curves for a red and a blue supergiant progenitor. The red supergiant is a $20 M_{\odot}$ star without mass lass; the blue supergiant had an initial mass of $17 M_{\odot}$ and accreted $3 M_{\odot}$ after its main-sequence phase. The explosion energy is $10^{51}$ erg in each case. 